\title{
Erratum \\ Reflecting on the institutional processes for college success: The experiences of four Chicanos in the context of inequality
}

Latino Studies (2013) 11, 624. doi:10.1057/lst.2013.47

Correction to: Latino Studies (2013) 11, 411-427. doi: 10.1057/lst.2013.24

Owing to a production error, the affiliation for the first author, Louie F. Rodríguez, was incorrectly listed as "California State University, Upland." This should have read "California State University, San Bernardino." The publishers apologize for this error. 